\title{
Graphene oxide-reinforced biodegradable genipin-cross-linked chitosan fluorescent biocomposite film and its cytocompatibility
}

This article was published in the following Dove Press journal:

International Journal of Nanomedicine

30 August 2013

Number of times this article has been viewed

\author{
Jianhua $\mathrm{Li}^{\prime}$ \\ Na Ren' \\ Jichuan Qiu' \\ Xiaoning $\mathrm{Mou}^{2}$ \\ Hong Liu' ${ }^{1,2}$ \\ 'Center of Bio and Micro/Nano \\ Functional Materials, State Key \\ Laboratory of Crystal Materials, \\ Shandong University, Jinan, People's \\ Republic of China; ${ }^{2}$ Beijing Institute \\ of Nanoenergy and Nanosystems, \\ Chinese Academy of Sciences, Beijing, \\ People's Republic of China
}

\begin{abstract}
A genipin-cross-linked chitosan/graphene oxide (GCS/GO) composite film was prepared using a solution casting method. Fourier transform infrared (FTIR) and ultravioletvisible (UV-Vis) spectroscopy of the composite films showed that the interactions between the $\mathrm{CS}$ and oxygen-containing groups of GO resulted in good dispersion of the GO sheets in the CS network. The addition of GO decreased the expansion ratio of the composite films in physiological conditions and increased the resistance to degradation by lysozymes in vitro. As well, the tensile strength values of the GCS/GO films were significantly increased with the increasing load of GO. Moreover, the GCS/GO composite film also maintained the intrinsic fluorescence of GCS. The in vitro cell study results revealed that the composite films were suitable for the proliferation and adhesion of mouse preosteoblast (MC3T3-E1) cells. The GCS/GO biocomposite films might have a potential use in tissue engineering, bioimaging, and drug delivery.
\end{abstract}

Keywords: chitosan film degradation, fluorescence, cytocompatibility

\section{Introduction}

Graphene, a one-atom-thick layer of sp2-bonded carbon atoms, has been extensively studied due to its extraordinary mechanical, thermal, and electronic properties. ${ }^{1,2}$ The cost-effective and large-scale production of graphene is usually achieved by the reduction of its precursor, graphite oxide, which is a layered material produced by the oxidation of graphite. ${ }^{3,4}$ Recently, graphene oxide (GO) has shown great promise for biomedical applications, such as drug delivery, ${ }^{5-7}$ cell imaging, ${ }^{5,8}$ enzyme immobilization, ${ }^{9,10}$ biosensors, ${ }^{11,12}$ etc. In contrast to pristine graphene, GO is rich in hydrophilic functional groups, such as $-\mathrm{COOH}$ and $-\mathrm{OH}$, which can significantly alter the van der Waals interactions and which make it easily dispersed in aqueous solutions and some organic solution. ${ }^{13}$ Thus, GO possesses strong interfacial interaction with polymers, especially hydrophilic ones. Therefore, there has recently been an attempt to use GO as a two-dimensional (2D) filler for the preparation of mechanically enhanced polymer composites. $^{14-16}$

Chitosan (CS), a biodegradable, nontoxic, antibacterial, and biocompatible naturally derived polymer, has attracted significant interest in a broad range of applications, such as food packaging, water treatment, separation membrane technology, tissue engineering, and drug delivery. ${ }^{17,18}$ Moreover, CS has a hydrophilic surface, promoting cell adhesion, proliferation, and differentiation and evokes a minimal foreign body reaction on implantation; therefore, CS-based biomaterials have been widely studied for biomedical application. ${ }^{17} \mathrm{CS}$-based films, for instance, have been used in stent insertion, ${ }^{19} \mathrm{drug}$ release,${ }^{20}$ and wound dressing..$^{21,22}$ However, the relatively weak mechanical properties
Correspondence: Hong Liu State Key Laboratory of Crystal Materials, Shandong University, 27 South Shanda Road, Jinan, People's Republic of China Tel +86 53I 88362807 Fax +8653I88362807 Email hongliu@sdu.edu.cn 
of CS and its ready swelling in water restrict its use in a wider application. Cross-linking is a convenient solution to solve this problem, allowing the creation of a three-dimensional (3D) cross-linked network matrix. Genipin, a naturally occurring and nontoxic cross-linking reagent, has been extensively used to obtain physically strong and biocompatible cross-linked products. ${ }^{23-25}$ By using genipin, the mechanical properties and the speed of degradation of CS can be controlled. ${ }^{24,25}$ In addition, CS-genipin conjugates possess a novel intrinsic fluorescent property, which broadens their use in biomedical applications, like in situ observation. ${ }^{26,27}$ In our previous work, we fabricated a hydroxyapatite-coated genipin-CS conjugation scaffold with fluorescent properties, which could provide effective ways for imaging the scaffold-cell interface and for tracing the adhesion, migration, and proliferation of cells, by a confocal laser scanning microscope (CLSM). ${ }^{26}$

Due to their individual excellent properties, both GO incorporated into CS and genipin-cross-linked CS (GCS) are expected to hold promising performance. Recently, Pan et al presented a simple approach to the fabrication of $\mathrm{GO} / \mathrm{CS}$ nanocomposite films with enhanced mechanical properties. ${ }^{28}$ In a similar work, Yang et al found that the incorporation of only $1 \mathrm{wt} \% \mathrm{GO}$ into CS composites dramatically increased their tensile strength and Young's modulus, by $122 \%$ and $64 \%$, respectively and that the glass-transition temperature increased as well. ${ }^{29}$ Here and in other excellent studies on CS/GO composites, ${ }^{16,30} \mathrm{GO}$ was demonstrated to be an efficient nanofiller for CS, and it can be expected that with largely improved mechanical properties, CS may play a more important role as biomaterials. Although the GO-reinforced CS materials synthesized in previous work are claimed to be useful as biomaterials, little work investigating the biodegradability or biocompatibility of GO-incorporated CS composites has been reported. In this study, we proposed a GCS/GO composite film, prepared using a solution casting method. In this composite, the mechanical properties, biodegradation, and swelling behavior of the film could be fine-tuned by adjusting the content of GO. Further, the intrinsic fluorescence caused by the cross-linkage of genipin still remained after adding GO to the CS matrix. Most importantly, an in vitro cell culture with the samples proved that this high-strength, fluorescent, biodegradable composite biofilm had very good cytocompatibility for biomedical application.

\section{Materials and methods Materials}

Medium molecular weight CS (Cat No 448877) (75\%-85\% deacetylated) and lysozyme (Cat No 62971) (70000 U/mg) were purchased from Sigma-Aldrich Corp (St Louis, MO, USA). Graphite flake (natural, -325 mesh, 99.8\%) was obtained from Alfa Aesar (Ward Hill, MA, USA). Genipin (99.0\% pure) was purchased from Linchuan Zhixin BioTechnology Co, Ltd (Jiangxi, People's Republic of China). The other reagents were bought from Sinopharm Chemical Reagent Co, Ltd (Shanghai, People's Republic of China).

\section{Preparation of genipin-cross-linked chitosan/graphene oxide composite film}

First, GO was prepared from graphite using an improved synthesis method. ${ }^{31}$ Briefly, a mixture of concentrated $\mathrm{H}_{2} \mathrm{SO}_{4} /$ $\mathrm{H}_{3} \mathrm{PO}_{4}(360: 40 \mathrm{~mL})$ was added to a mixture of $3.0 \mathrm{~g}$ graphite flakes and $18.0 \mathrm{~g} \mathrm{KMnO}_{4}$. The reaction was heated to $50^{\circ} \mathrm{C}$ and stirred for 12 hours, then cooled to room temperature and poured onto $\sim 350 \mathrm{~g}$ ice chips. Then, $3.0 \mathrm{~mL}$ of $30 \% \mathrm{H}_{2} \mathrm{O}_{2}$ was slowly added to obtain a bright yellow dispersion. The suspension was then centrifuged (4000 rpm for 4 hours), and the supernatant was decanted away. The obtained deposit was washed repeatedly with deionized water and then dialyzed (molecular weight cutoff [MWCO] 3000) for 2 weeks to obtain a clean dispersion of GO. Finally, the GO suspension was diluted to certain concentrations and sonicated to achieve single-layered sheets.

CS powder (1 g) was added into $50 \mathrm{~mL}$ of aqueous solution containing different amounts of GO. The mixture was then dissolved by the dropwise addition of $800 \mu \mathrm{L}$ acetic acid. After stirring overnight, a transparent and welldispersed CS/GO nanocomposite suspension was obtained. ${ }^{29}$ To obtain the cross-linked CS matrix (GCS), $100 \mu \mathrm{L}$ genipin solution $(50 \mathrm{mg} / \mathrm{mL}$ in ethanol) was then added and stirred for 30 minutes at room temperature. The solution was then carefully poured into a $10 \mathrm{~mm}$ petri dish and incubated at $30^{\circ} \mathrm{C}$ overnight to form a film, followed by drying in a vacuum oven at $40^{\circ} \mathrm{C}$ for 48 hours. CS/GO composite films without cross-linking were obtained by following the same procedures but with no addition of genipin. By changing the weight fraction of the GO added to the GCS, such as $0 \%$, $0.1 \%, 0.5 \%, 1 \%$, and $2 \%$, a series of samples, referred to as GCS, GCS/GO-0.1, GCS/GO-0.5, GCS/GO-1, and GCS/ GO-2, were prepared.

\section{Characterization}

As-synthesized GO sheets were characterized by atomic force microscopic (AFM) (Dimension Icon ${ }^{\circledR}$; Bruker Corp, Billerica, MA, USA) measurements. The samples were prepared for AFM by dropping dilute GO solution onto a 
fresh-peeled mica sheet, followed by drying in a clean oven. $\mathrm{X}$-ray photoelectron spectroscopic (XPS) measurements were carried out on a Thermo Fisher Scientific Escalab 250 spectrometer (Thermo Fisher Scientific, Inc, Waltham, MA, USA) with monochromatized $\mathrm{Al} \mathrm{K} \alpha$ excitation, and $\mathrm{C} 1 \mathrm{~s}(284.5 \mathrm{eV})$ was used to calibrate the peak positions of the elements.

The stability of the suspension of GO/CS in aqueous acetic acid solution (without dilution) was characterized with a Zeta Potential Analyzer (ZetaPALS; Brookhaven Instruments Corp, Holtsville NY, USA). The ultraviolet-visible (UV-Vis) diffuse reflectance spectra of the obtained films samples were determined on a Shimadzu UV 2550 spectrophotometer (Shimadzu Corp, Kyoto, Japan) equipped with an integrating sphere. Fourier transform infrared (FTIR) spectra were recorded using an infrared (IR) spectrophotometer (Nicolet Nexus 670; Thermo Fisher Scientific, Inc). The spectrum was obtained with 64 scans per sample ranging from $4000-400 \mathrm{~cm}^{-1}$, with a resolution of $4 \mathrm{~cm}^{-1}$. The morphologies of the GCS and GCS/GO films were imaged using a scanning electron microscope (SEM) (Hitachi S-4800; Hitachi Ltd, Tokyo, Japan), at an accelerating voltage of $10 \mathrm{kV}$. Samples for cross-sectional view were obtained by tensile fracture. All samples were sputtered with gold before observation.

To investigate how the GO sheets influenced the swelling of the composite films, three samples from each group were immersed in phosphate-buffered saline (PBS) $(50 \mathrm{mM}$, pH 7.4) until their weight became constant. The samples were then removed from the PBS, and their surfaces were blotted with filter paper before being measured. The expansion ratio of the lateral length of the GCS/GO film was calculated according to the following formula:

$$
\text { Expansion ratio }=\left(\mathrm{L}-\mathrm{L}_{0}\right) / \mathrm{L}_{0}
$$

where $\mathrm{L}_{0}$ and $\mathrm{L}$ were the lateral length of GCS/GO film before and after soaking in PBS, respectively.

For the in vitro enzymatic degradation study, the initial films were placed in $10 \mathrm{~mL}$ vials containing $5 \mathrm{~mL}$ of PBS (0.1 M, pH 7.4) containing $2 \mathrm{mg} / \mathrm{mL}$ lysozyme (70,000 U/mg; Sigma-Aldrich Corp) and $0.1 \mathrm{mg} / \mathrm{mL} \mathrm{NaN}_{3}$. The vials were stored in an incubator set at $37^{\circ} \mathrm{C}$ and agitated at $100 \mathrm{rpm}$. Three samples of each group were weighed before in vitro degradation test $(\mathrm{Wb})$ and collected at fixed time intervals and rinsed thoroughly with deionized water. Samples were then dried at room temperature and weighed to obtain the remaining weights $(\mathrm{Wr})$. The percentage of remaining weight of the CS film was calculated after the enzymatic degradation using the formula:

Percentage of remaining weight $\%=\mathrm{Wr} / \mathrm{Wb} \times 100 \%$

The tensile properties of the composite films were measured using an automatic mechanical testing machine (WDW-1; Zhixing Test, Jinan, People's Republic of China) fitted with a $100 \mathrm{~N}$ load cell at room temperature. The strain rate was $5.0 \mathrm{~mm} / \mathrm{min}$. The thickness of all tested samples was determined by SEM.

The fluorescence emission spectra of GCS/GO films were measured using a fluorescence spectrometer (FLS920; Edinburgh Instruments Ltd, Livingston, UK) at the excitation of $488 \mathrm{~nm}$. The samples used for wide-field fluorescence microscopy were rinsed in PBS and observed using an Olympus IX $71{ }^{\circledR}$ inverted microscope (Olympus Corporation, Tokyo, Japan) coupled with a charge-coupled device (CCD) and display controller software. According to the obtained emission spectra, the fluorescence images were recorded through U-MNIBA3 $(\lambda \mathrm{ex}=460-490 \mathrm{~nm}$; $\lambda \mathrm{em}>510 \mathrm{~nm})$ and U-MWG2 $(\lambda \mathrm{ex}=510-550 \mathrm{~nm} ; \lambda \mathrm{em}$ $>570 \mathrm{~nm}$ ) filter combinations. The International Organization for Standardization (ISO) sensitivity was set at 800 , and the exposure time at $100 \mathrm{msec}$ for U-MNIBA3 and $10 \mathrm{msec}$ for U-MWG2.

\section{Cell culture}

The mouse preosteoblast cell line (MC3T3-E1) used in this study was obtained from the Cell Bank of Type Culture Collection of Chinese Academy of Sciences, Shanghai, People's Republic of China. All samples $(5 \times 5 \mathrm{~mm})$ were sterilized by exposing each side of the sample to UV light for 30 minutes, washed three times in sterile PBS, and presoaked in 48-well-plates with cell culture medium overnight, before cell seeding. After removing the medium, $30 \mu \mathrm{L}$ of the cell suspension (containing 3000 cells) was gently injected onto each sample. Care was taken that the cells were seeded on the top surface of the film, without any spillage. Subsequently, the cell-seeded constructs were incubated for 20 minutes so that the cells were allowed to adhere. Finally, $400 \mu \mathrm{L}$ of $\alpha$-minimal essential medium (a-MEM) (Cat No SH30265.01B, Thermo Scientific HyClone, Beijing, People's Republic of China), supplemented with 10\% fetal bovine serum (FBS) (Gibco; Cat No 10099-141, Life Technologies Corp) and 1\% penicillin and streptomycin, was gently injected into each well. Cells were cultured in a humidified atmosphere of $5 \% \mathrm{CO}_{2}$ at $37^{\circ} \mathrm{C}$. The culture medium was changed every 2 days.

\section{Cell viability assay}

A Cell Counting Kit-8 (CCK-8; Dojindo Molecular Technology, Kumamoto, Japan) was employed to quantitatively 
evaluate the cell viability of various samples after cultivation for 1, 3, and 5 days. 2-(2-methoxy-4-nitrophenyl)-3(4-nitrophenyl)-5-(2, 4-disulfophenyl)-2 H-tetrazolium monosodium salt (water-soluble tetrazolium salt [WST8]) was used as a substrate. First, each tested sample was removed to a new 48 -well plate filled with $360 \mu \mathrm{L}$ of serumfree $\alpha$-MEM medium plus $40 \mu \mathrm{L}$ of CCK- 8 solution per well. After 4 hours incubation at $37^{\circ} \mathrm{C}$, the resultant production of water-soluble formazan dye was assayed at a wavelength of $450 \mathrm{~nm}$ by a microplate reader (Multiscan MK3; Thermo Fisher Scientific, Inc). The 48-well tissue culture plate was adopted as a negative control. Three parallel replicates were used for each sample.

Cell viability was then further assessed using a LIVE/ DEAD $^{\circledR}$ Viability/Cytotoxicity Kit (Invitrogen ${ }^{\text {TM }}$ \# L-3224; Life Technologies, Corp). The kit can quickly discriminate live from dead cells by simultaneously staining with green-fluorescent acetomethoxy calcein ester (calceinAM) to indicate intracellular esterase activity and redfluorescent ethidium homodimer-1 to indicate the loss of plasma membrane integrity. First, the staining solution was prepared by adding $1 \mu \mathrm{L}$ calcein-AM and $2.5 \mu \mathrm{L}$ ethidium homodimer- 1 solution to $1 \mathrm{~mL}$ PBS. On day 1 and day 5 , the culture medium was removed, and the samples were washed gently with PBS. Then $200 \mu \mathrm{L}$ of Live/Dead stain was added per well and incubated for 10 minutes at $37^{\circ} \mathrm{C}$. Finally, the samples were washed with PBS and observed using a CLSM (model number TCS SP2) (Leica Microsystems, Wetzlar, Germany) at an excitation wavelength of $488 \mathrm{~nm}$ (green) and $533 \mathrm{~nm}$ (red).

\section{Statistics}

Data were reported as mean plus standard deviation. Comparative studies of the means were performed by analysis of variance (ANOVA), using SPSS for Windows, Version 13.0 (SPSS Inc, Chicago, IL, USA) for multiple comparisons, and statistical significance was accepted at $P<0.05$.

\section{Results and discussion}

\section{Film composition and structure}

The size and thickness information for GO sheets were observed by AFM (Figure 1A). The measured thickness of the GO sheets was uniform $(\sim 1.1 \mathrm{~nm})$, showing that the GO sheets were fully exfoliated into individual ones
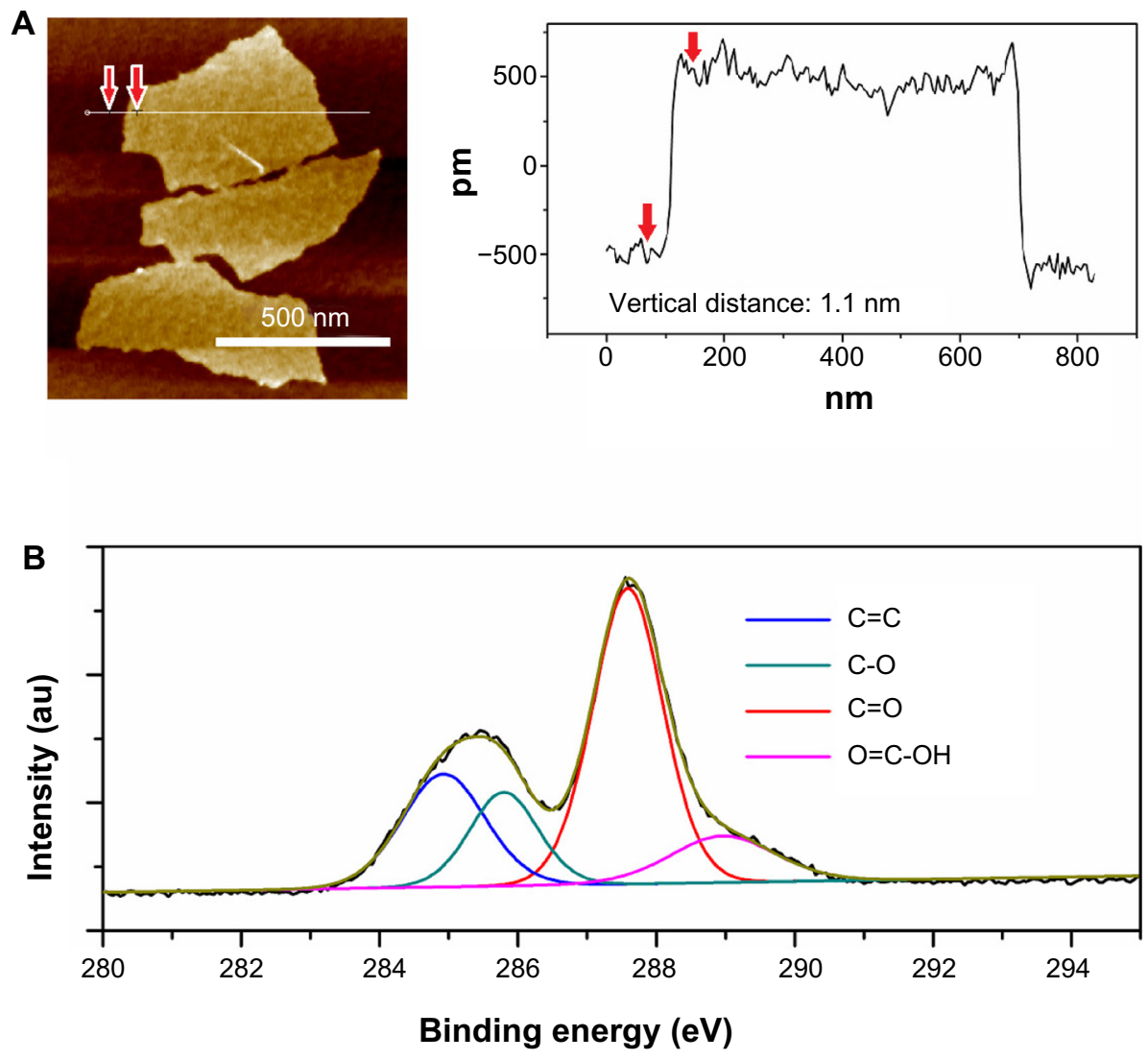

Figure I AFM image with height profiles (A) and XPS CIs spectra (B) of GO sheets.

Note: The black curve is obtained data, the brown one is the corresponding fitting curve.

Abbreviations: AFM, atomic force microscope; GO, graphene oxide; XPS, X-ray photoelectron spectroscope. 
in water by ultrasonic treatment. From the $\mathrm{C} 1 \mathrm{~s}$ spectra of GO shown in Figure 1B, the $\mathrm{C} 1 \mathrm{~s}$ band can be fitted into four peaks that correspond to the following functional groups: carbon sp2 $(\mathrm{C}=\mathrm{C}, 284.5 \mathrm{eV})$, epoxy/hydroxyls $(\mathrm{C}-\mathrm{O}, 285.8 \mathrm{eV})$, carbonyl $(\mathrm{C}=\mathrm{O}, 287.4 \mathrm{eV})$, and carboxylates $(\mathrm{O}=\mathrm{C}-\mathrm{O}, 289.0 \mathrm{eV}) .{ }^{3}$ These abundant hydrophilic oxygen functional groups made GO highly soluble in aqueous solution.

After mixing with CS solution by vigorous stirring, the suspension showed good stability over a period of several months (Figure 2A). The zeta potential value of $\mathrm{GO} / \mathrm{CS}$ dispersion $(\sim \mathrm{pH}=2)$ was around $30 \mathrm{mV}$ (Table 1), indicating a metastable dispersion of GO sheets in CS solution. Such a good dispersion of GO sheets in CS solution may be the result of the hydrophilic groups in $\mathrm{GO}$ and electrostatic interaction between the cationic CS and the negative charge on the surface of the GO.

To investigate the interaction between CS and GO sheets as well as the interaction between GCS and the GO sheets, the UV-Vis absorbance spectra of the CS/GO and GCS/GO films were obtained, shown in Figure 2C. For the original CS, a strong absorption band was evident around $250 \mathrm{~nm}$, which was caused by the $\mathrm{n} \rightarrow \sigma^{*}$ transition for the amino groups of $\mathrm{CS}^{32}$ After incorporating the GO, a clear red shift toward longer wavelength, around $320 \mathrm{~nm}$, could
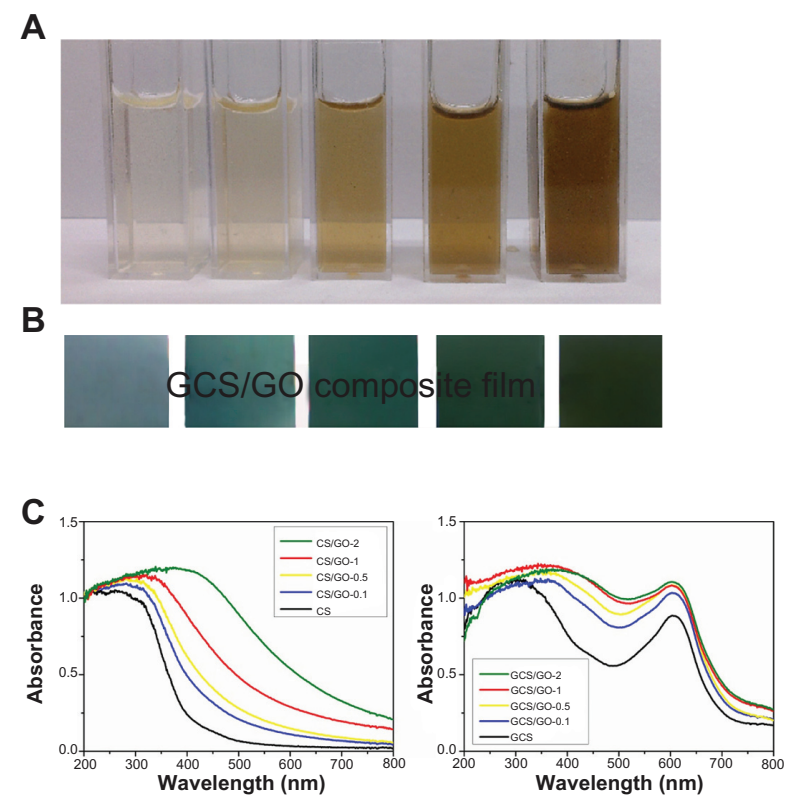

Figure 2 Photographs of neat CS dissolved in acetic acid, and 0.1 wt\%, 0.5 wt\%, I wt\%, and 2 wt\% GO/CS dispersions (from left to right), before cross-linking (A). Photographs of the GCS, GCS/GO-0.I, GCS/GO-0.5, GCS/GO-I and GCS/GO-2 composite films (from left to right) (B). UV-Vis diffuse reflectance spectra of the cross-linked (GCS/GO) and non-cross-linked (CS/GO) composite films (C).

Abbreviations: CS, chitosan; GCS, genipin-cross-linked chitosan; GO, graphene oxide; UV-Vis, ultraviolet-visible.
Table I Zeta potential measurement of the CS/GO dispersions

\begin{tabular}{lllllll}
\hline Sample & CS & $\begin{array}{l}\mathbf{0 . 1} \text { wt\% } \\
\text { GO in } \\
\text { CS }\end{array}$ & $\begin{array}{l}\mathbf{0 . 5} \text { wt\% } \\
\text { GO in } \\
\text { CS }\end{array}$ & $\begin{array}{l}\text { I wt\% } \\
\text { GO in } \\
\text { CS }\end{array}$ & $\begin{array}{l}\mathbf{2} \text { wt\% } \\
\text { GO in } \\
\text { CS }\end{array}$ & GO \\
\hline $\begin{array}{l}\text { Zeta potential } \\
(\mathrm{mV})\end{array}$ & 76.5 & 31.3 & 32.1 & 30.3 & 27.8 & -56.1 \\
\hline
\end{tabular}

Abbreviations: CS, chitosan; GO, graphene oxide.

be seen, which was due to the ground-state electron donoracceptor interaction between the two components. Then, the genipin was introduced to the CS/GO system, and the absorption peak further shifted to $\sim 370 \mathrm{~nm}$ due to the conversion of primary amine groups on CS to secondary amide linkages between CS and genipin. ${ }^{33}$ The characteristic absorption peak at $\sim 605 \mathrm{~nm}$, which corresponds to genipin-cross-linking-formed blue pigment, ${ }^{34}$ remained at the original position but increased the absorption with the addition of GO. The increase in absorbance led to a change in the color of the GCS/GO film from bluish to dark-blue (Figure 2B).

To particularly identify the interaction between GO and CS, an FTIR study of the CS/GO film was carried out. The obtained spectra are shown in Figure 3. The peaks at 1080, 1423 , and $1634 \mathrm{~cm}^{-1}$ correspond to $\mathrm{C}-\mathrm{O}-\mathrm{C}$ stretching vibrations, $\mathrm{C}-\mathrm{OH}$ stretching, and the $\mathrm{C}-\mathrm{C}$ stretching mode of the sp2 carbon skeletal network, respectively, while peaks located at 1734 and $3280 \mathrm{~cm}^{-1}$ correspond to $\mathrm{C}-\mathrm{O}$ stretching vibrations of the $-\mathrm{COOH}$ groups and $\mathrm{O}-\mathrm{H}$ stretching vibration, respectively. These functional groups make GO highly hydrophilic and render it dispersible. In the spectrum of CS, there were two characteristic absorbance bands centered at 1650 and $1591 \mathrm{~cm}^{-1}$, which corresponded to the

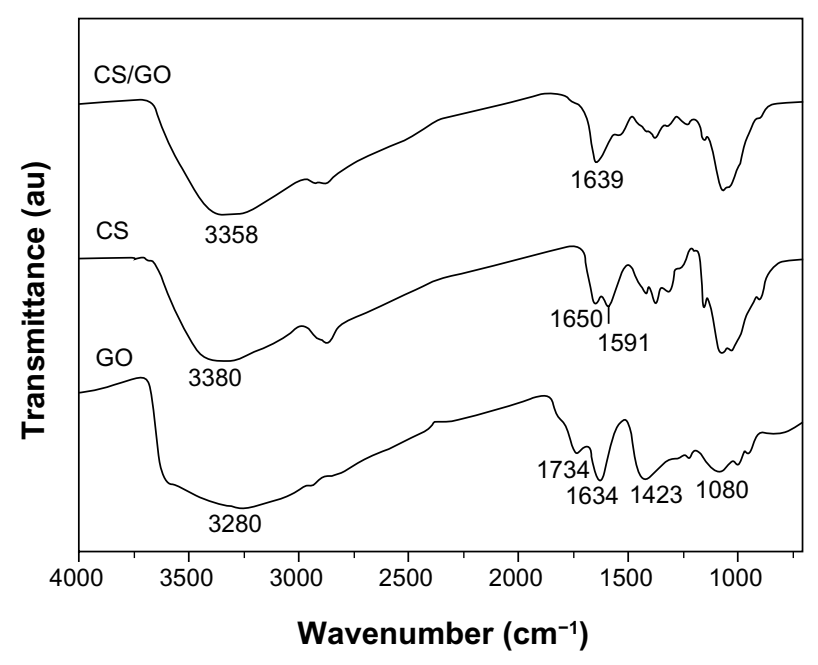

Figure 3 FTIR spectra of GO, pure CS, and CS/GO (2 wt\%) composite film. Abbreviations: CS, chitosan; FTIR, Fourier transform infrared; GO, graphene oxide. 
$\mathrm{C}=\mathrm{O}$ stretching vibration of amide $\mathrm{I}$ and the $\mathrm{N}-\mathrm{H}$ bending of $-\mathrm{NH}_{2}$, respectively. The FTIR spectrum of the CS/GO composite showed a combination of characteristics similar to that of the pristine CS and GO. In the spectrum of CS, peaks corresponding to $\mathrm{NH}_{2}$ absorbance vibration are at $1591 \mathrm{~cm}^{-1}$. In the spectrum of $\mathrm{GO}$, peaks corresponding to $\mathrm{C}=\mathrm{O}$ stretch of carboxylic group is at $1734 \mathrm{~cm}^{-1}$. In the spectrum of CS/ $\mathrm{GO}$, both of the above peaks disappeared, which suggested that interactions existed between CS and GO. Moreover, the $\mathrm{C}=\mathrm{O}$ characteristic stretching band of the amide at $1650 \mathrm{~cm}^{-1}$ was downshifted due to the synergistic effect of hydrogen bonding between CS and the oxygenated groups in GO and the electrostatic interaction between polycationic $\mathrm{CS}$ and the negative charge on the surface of GO (Figure S1). ${ }^{29}$ These interactions may have a strong impact on the mechanical properties, swelling, and degradation of the composite film.

The SEM images of the as-prepared samples clearly showed how the GO sheets were arranged in the CS (Figures 4 and S2). As shown in Figure 4A and B, the cross-sectional surface of the GCS sample was very smooth. However, the cross-section of the GCS/GO sample had a layered structure (Figure 4D), in which the GO sheets seemed parallel to each other and tightly enwrapped by the CS, and no exposed graphene sheet could be found (Figure 4D). This indicates that the GO sheets had a strong interaction with the surrounding CS molecules. The film obtained in this study was prepared by the solution casting method. The 2D GO sheets naturally lay down when the water slowly dried out, resulting in the parallelaligned structure, similar to that described in the previous report. ${ }^{28}$ The layered structure of the GCS/GO film, together with the firm interactions between those two opponents, may have had a positive influence on the physical properties.
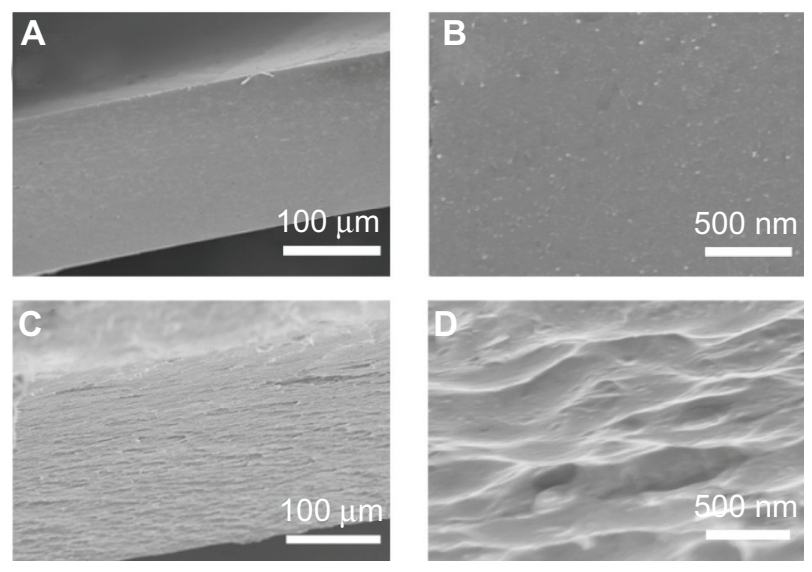

Figure 4 Typical cross-sectional SEM images of GCS (A and B) and GCS/GO-I (C and D) samples, and their enlarged views (B and $\mathbf{D})$.

Abbreviations: GCS, genipin-cross-linked chitosan; GO, graphene oxide; SEM, scanning electron microscope.

\section{Swelling and degradation}

$\mathrm{CS}$, containing hydroxyl and amino groups, is readily hydrated in water because water molecules strongly interact with these hydrophilic groups, resulting in weakening of the intermolecular hydrogen bonding. From the swelling results (Figure 5), the expansion ratio of the samples depended directly on the GO content. From GCS/GO- 0.1 to GCS/GO-2, the expansion ratio decreased from 1.60 to 1.27 , while for the GCS sample, the expansion ratio reached 1.65 . The reduction in expansion ratio may be attributed to the decrease in the content of the free amino and hydroxyl groups of CS after the addition of GO. The GO sheets bridged the neighboring CS molecules by forming new hydrogen bonding, leading to a pinning effect to the CS matrix (Figure S1). With the increase of GO content, the CS matrix became more compact, with less space for water containment and decrease in the expansion ratio. As reported, genipin can also change the hydrophilicity of CS by changing the reaction time and concentration. ${ }^{35}$ In this study, the concentration of genipin and the cross-linking time were fixed at appropriate values in all the samples, so the influence on swelling from the genipin could be ignored, and the GO content was the only variable. Therefore, increasing the loading amount of GO decreased the expansion ratio of the the GCS film. This observation can provide a new strategy for controlling the swelling behavior of hydrophilic polymers, ie, use of the $2 \mathrm{D}$ building block, whereas to date, most methods have focused on cross-linking with small chemical molecules. Moreover, the shape retention of the GCS/GO film may prove beneficial for use as a stent, heart valve, or skin scaffold.

The degradation of a biomaterial is very important for its biomedical use. For instance, tissue engineering, which aims at the regeneration of new tissues, requires biomaterials to be degradable and absorbable with a proper rate that matches the speed of new tissue growth. The obtained films were further characterized by an in vitro investigation of their resistance to enzymatic degradation. It is known that CS can be degraded by lysozyme, a body enzyme with concentration in human serum of about $1.5 \mu \mathrm{g} / \mathrm{mL} .{ }^{36}$ For convenience, a higher concentration of lysozyme was employed in the current study. As shown in Figure 6, the samples without GO degraded faster than those with GO. With increasing loading content of GO, the composite film showed a greater resistance to enzymatic degradation. According to the swelling study, the expansion ratio of GCS/GO film was dependent on the GO amount. Therefore, the decreased expansion ratio resulted in less enzyme content penetrating into the CS matrix and thus, a slowing down of the degradation rate. Further, it has been found that lysozyme can be spontaneously immobilized 


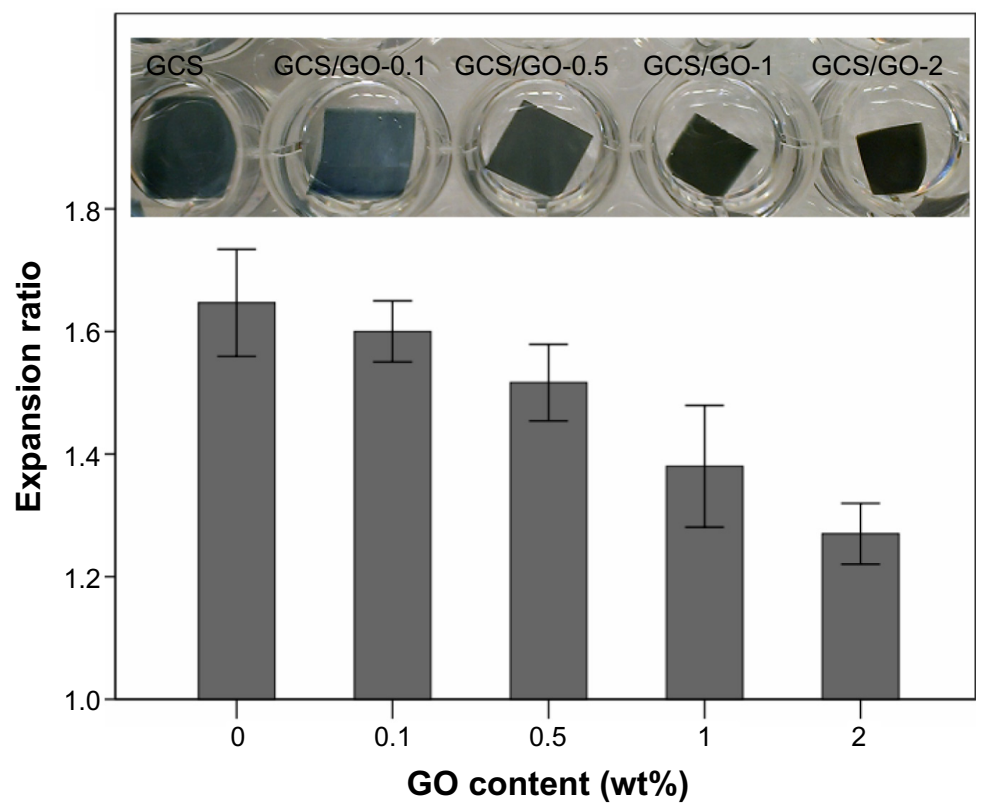

Figure 5 Expansion ratio of the GCS films with different GO content, in PBS.

Abbreviations: GCS, genipin-cross-linked chitosan; GO, graphene oxide; PBS, phosphate-buffered saline.

on GO sheets because GO sheets have abundant oxygencontaining groups, which enable enzyme immobilization; ${ }^{9}$ thus, the GO-dependent degradation of GCS might partially have been due to the immobilization of lysozyme on the GO sheets. For the above reasons, the addition of GO might be a potential method to adjust the degradation of GCS film and other polymers, to enhance their suitability for tissue engineering and long-acting drug delivery.

\section{Mechanical test}

Figure 7 shows the tensile study results of the GCS/GO composite films with different GO content. Compared with the GCS film, the tensile strength of the composite films increased

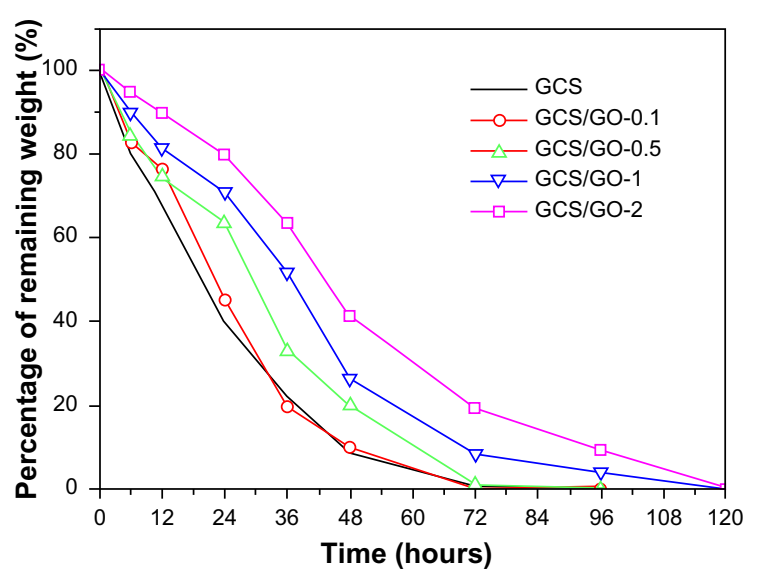

Figure 6 Percentage of remaining weight of GCS with different GO loading content, after enzymatic degradation.

Abbreviations: GCS, genipin-cross-linked chitosan; GO, graphene oxide. gradually with the increasing loading of $\mathrm{GO}$, as shown in Figure 7. With GO loading from $0.1 \mathrm{wt} \%$ to $1 \mathrm{wt} \%$, the tensile strength values increased from $\sim 70 \mathrm{MPa}$ to $\sim 110 \mathrm{MPa}$. This indicates that the GO sheets could significantly improve the mechanical properties of the GCS films. For the GCS/GO-2 samples, the tensile strength slightly decreased, which may have been due to the aggregation of GO sheets. According to the previous reports, ${ }^{28,37}$ the same results were found in CS/ GO composite film. Most importantly, our study demonstrates that the GO sheets can also enhance the tensile strength of the genipin-cross-linked CS network.

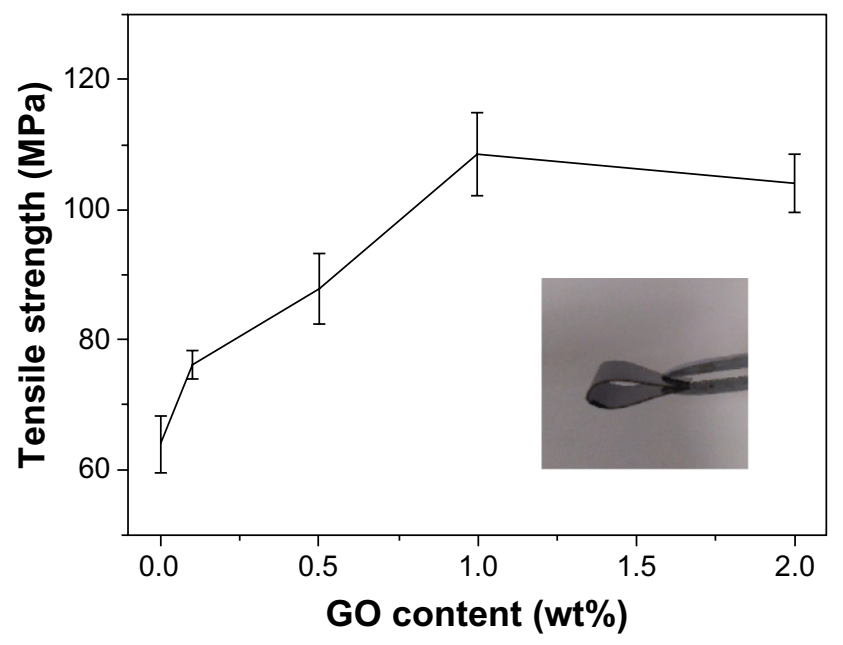

Figure 7 Tensile strength of GCS/GO composite films with different GO content. Note: Insert photo shows that the GCS/GO composite film can be bent to a large angle, indicating its flexibility.

Abbreviations: GCS, genipin-cross-linked chitosan; GO, graphene oxide. 


\section{Fluorescence study}

It has been found that genipin reacts with primary amine groups on CS, via a nucleophilic attack to open the dihydropyran ring, forming nitrogen-containing heterocyclic rings ${ }^{38}$ It appears that a large conjugated system, possibly the $\pi-\pi^{*}$ conjugation, is formed by this reaction, thus resulting in the fluorescence in the cross-linked CS. ${ }^{39}$ In our study, genipin was added after the GO sheets were blended into the CS solution. Both the GO and the genipin could react with primary amine group, therefore, there would have been a lesser amount of primary amine groups available for genipin after the addition of GO. Further, the welldispersed GO sheets divided the large conjugated system into smaller ones. Therefore, the fluorescence intensity of the composite films decreased with increasing loading of GO (Figure 8A). Another explanation for the decreasing fluorescence intensity may be that the GO sheets could have blocked or reduced the intensity of the excitation light as well as the emission light. It should also be noted that chemically derived GO also exhibits weak blue to ultraviolet fluorescence (centered around $390 \mathrm{~nm}$, for thin film) when excited with ultraviolet radiation. ${ }^{40,41}$ However, the fluorescence intensity is so weak (below 3 kilocounts per second for neat GO thin film) compared with genipin-generated fluorescence that the fluorescence from GO can be ignored due to its low weight fraction.

A
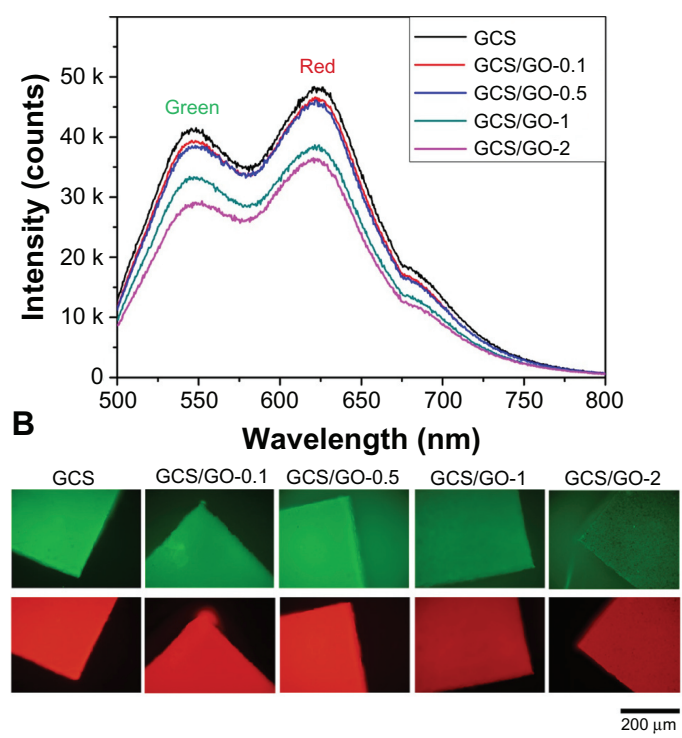

Figure 8 Fluorescence emission spectra (A) and wide-field fluorescence microscopy images (B) of the GCS/GO composite with different GO loadings.

Notes: Green images were recorded through U-MNIBA3 $(\lambda$ ex $=460-490 \mathrm{~nm}$; $\lambda \mathrm{em}>510 \mathrm{~nm})$ and red ones through U-MWG2 $(\lambda \mathrm{ex}=510-550 \mathrm{~nm} ; \lambda \mathrm{em}>570 \mathrm{~nm})$ filter combinations.

Abbreviations: GCS, genipin-cross-linked chitosan; GO, graphene oxide.
As shown in Figure 8A, the composite films could generate green and red emission light when excited at $488 \mathrm{~nm}$. Therefore, the film could be clearly visualized with the appropriate filter combinations, by wild field imaging (Figure 8B). It can be seen that the GO-incorporated samples were still highly visible, with reduced fluorescence intensity. This novel fluorescent property may further add to the advantages of using GCS for biomedical applications, such as bioimaging.

In the fluorescence image of the GCS/GO-2 sample, some aggregated GO sheets (black spots) can be clearly observed. Kim et al reported that graphene-based sheets can be made highly visible under a fluorescence microscope by quenching the emission from a dye coating, which can be conveniently removed afterward, by rinsing, without disrupting the sheets. ${ }^{42}$ Having interaction with GO sheets and intrinsic fluorescence, the GCS platform might be another potential method to immobilize and visualize graphene sheets under a fluorescence microscope.

\section{Biocompatibility}

The biocompatibility of the GCS and GCS/GO composite films with different GO content was assessed by in vitro culture with MC3T3-E1 cells on the films. The cell proliferation results are shown in Figure 9. The cell counting results revealed no visible reduction in viability between the control and experimental groups, at each time interval. On day 1 , the little variance between each group indicated there would be no difference in cell attachment on the

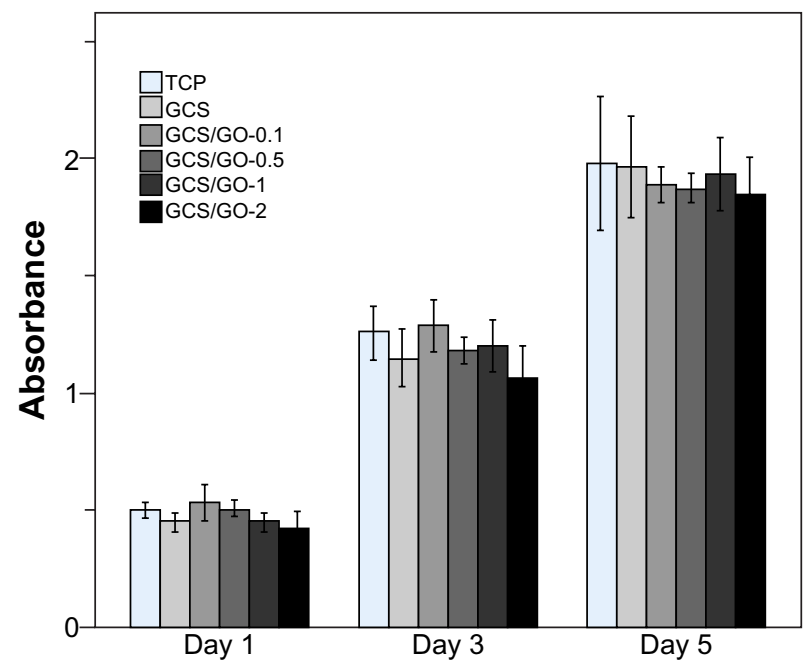

Figure 9 Cell-counting results of the TCP, GCS, and GCS/GO composite films on day $\mathrm{I}, 3$, and 5 .

Notes: The bars represent the standard deviations of three replications. Abbreviations: GCS, genipin-cross-linked chitosan; GO, graphene oxide; TCP, tissue culture plate (negative control). 

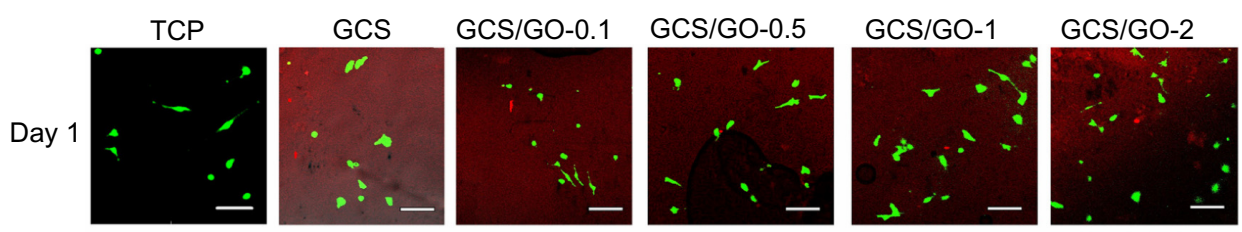

Day 4
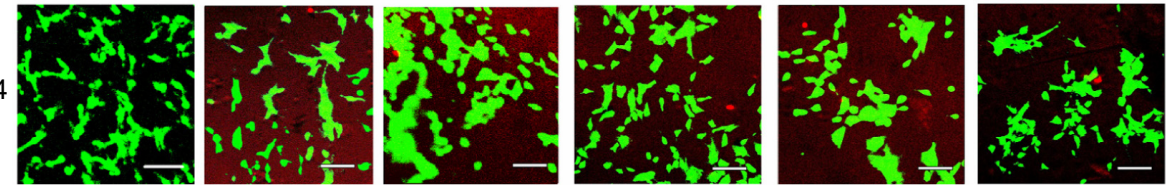

Figure 10 Fluorescent images of the MC3T3-EI cells-seeded TCP, GCS, and GCS/GO composite films on the first and fourth day of cultivation. The green represents living cells; the bright red represents dead cells; the dark red represents the chitosan film.

Note: $\mathrm{Bar}=50 \mu \mathrm{m}$.

Abbreviations: GCS, genipin-cross-linked chitosan; GO, graphene oxide; TCP, tissue culture plate (negative control).

different samples. On day 3 , the cell numbers in all groups were about 2.5 times greater than those on day 1 . On day 5 , there was no significant difference $(P>0.05)$ in the absorbance of the GCS/GO composite films compared with that of the pure GCS, showing that the addition of GO sheets into CS matrix had no negative effect on the cell growth. The observed results reveal that all the composite samples were suitable for the proliferation of the MC3T3-E1 cells, indicating good cytocompatibility.

To get visual evidence of the cytocompatibility of the GCS/GO composite films, MC3T3-E1 cells were seeded on the samples and observed under a fluorescent microscope, after staining the cells with a LIVE/DEAD assay, at days 1 and 4 of cultivation. In Figure 10, live cells are shown to have fluoresced green, and dead cells fluoresced bright red. The dark red background resulted from the fluorescent light of the GCS. From the fluorescent imaging, the proliferation of the seeded cells can be clearly seen. These results suggest that the addition of GO was not detrimental to the viability of the osteoblasts. As the content of GO increased, no obvious difference was seen in cell viability between the composite groups.

It has been reported that neat GO exhibits a dose-dependent toxicity to cells and animals. ${ }^{43}, 44$ Yet, the additional amount of GO has been at a low level in most graphene polymer composites, and therefore, most reported grapheneincorporated composites have showed good biocompatibility. For instance, graphene/CS composites were found to be biocompatible with L929 cells - the cells could adhere and grow on the composite films as well as on pure CS film. ${ }^{37}$ The polar GO has also been found to facilitate the cell attachment and proliferation of osteoblasts on hybrid CS network structures. ${ }^{16}$ Here, the in vitro cell culture with the GCS/GO composite sample has also proven nontoxic, which may be attributed to the excellent biocompatibility of $\mathrm{GO}$ at low concentration and the hydrophilic property of GO, as well as the biocompatible nature of GCS. Furthermore, biomolecules like enzyme and anticancer drugs can be further conjoined to the GO sheets, making the biocompatible GCS/GO composite a promising platform for biomedical use.

\section{Conclusion}

In conclusion, a GCS film conjoined with GO sheets with diverse novel properties was fabricated by a solution casting method. The well-dispersed GO sheets assembled in the GCS were found to reinforce the CS film by increasing the tensile strength and resistance to enzymatic degradation and by decreasing the expansion in aqueous solution. Most importantly, the GCS/GO film maintained the intrinsic fluorescence and the cytocompatibility of the GCS matrix. Taking advantage of the versatility of GO sheets, GCS/GO biocomposite films might have a potential use in a broader range of biomedical applications, such as tissue engineering, bioimaging, and drug delivery.

\section{Acknowledgments}

The authors are thankful for funds from National Natural Science Foundation of the People's Republic of China (NSFDYS: 50925205, 50990303, and 51002089), the Innovation Research Group (IRG: 51021062), the Program of Introducing Talents of Discipline to Universities in China (111 Program b06015), and the "100 Talents Program" of the Chinese Academy of Sciences.

\section{Disclosure}

The authors report no conflicts of interest in this work.

\section{References}

1. Geim AK, Novoselov KS. The rise of graphene. Nat Mater. 2007;6; 183-191. 
2. Castro Neto AH, Guinea F, Peres NMR, Novoselov KS, Geim AK. The electronic properties of graphene. Rev Mod Phys. 2009;81:109.

3. Stankovich S, Dikin DA, Piner RD, et al. Synthesis of graphene-based nanosheets via chemical reduction of exfoliated graphite oxide. Carbon NY. 2007;45:1558-1565.

4. Park S, Ruoff RS. Chemical methods for the production of graphenes. Nat Nanotechnol. 2009;4(4):217-224.

5. Sun X, Liu Z, Welsher K, et al. Nano-graphene oxide for cellular imaging and drug delivery. Nano Res. 2008;1(3):203-212.

6. Zhang L, Xia J, Zhao Q, Liu L, Zhang Z. Functional graphene oxide as a nanocarrier for controlled loading and targeted delivery of mixed anticancer drugs. Small. 2010;6(4):537-544.

7. Rana VK, Choi MC, Kong JY, et al. Synthesis and drug-delivery behavior of chitosan-functionalized graphene oxide hybrid nanosheets. Macromol Mater Eng. 2011;296(2):131-140.

8. Wang Y, Li Z, Hu D, Lin CT, Li J, Lin Y. Aptamer/graphene oxide nanocomplex for in situ molecular probing in living cells. $\mathrm{J} \mathrm{Am} \mathrm{Chem}$ Soc. 2010;132(27):9274-9276.

9. Zhang J, Zhang F, Yang H, et al. Graphene oxide as a matrix for enzyme immobilization. Langmuir. 2010;26(9):6083-6085.

10. Zhang Y, Zhang J, Huang X, Zhou X, Wu H, Guo S. Assembly of graphene oxide-enzyme conjugates through hydrophobic interaction. Small. 2012;8(1):154-159.

11. Wan Y, Wang Y, Wu J, Zhang D. Graphene oxide sheet-mediated silver enhancement for application to electrochemical biosensors. Anal Chem. 2011;83(3):648-653.

12. Song W, Li DW, Li YT, Li Y, Long YT. Disposable biosensor based on graphene oxide conjugated with tyrosinase assembled gold nanoparticles. Biosens Bioelectron. 2011;26(7):3181-3186.

13. Dreyer DR, Park S, Bielawski CW, Ruoff RS. The chemistry of graphene oxide. Chem Soc Rev. 2010;39:228-240.

14. Xu Y, Hong W, Bai H, Li C, Shi G. Strong and ductile poly(vinyl alcohol)/graphene oxide composite films with a layered structure. Carbon NY. 2009;47(15):3538-3543.

15. Wang $\mathrm{H}, \mathrm{Hao} \mathrm{Q}$, Yang $\mathrm{X}$, Lu L, Wang $\mathrm{X}$. Effect of graphene oxide on the properties of its composite with polyaniline. ACS Appl Mater Interfaces. 2010;2(3):821-828.

16. Depan D, Girase B, Shah JS, Misra RD. Structure-process-property relationship of the polar graphene oxide-mediated cellular response and stimulated growth of osteoblasts on hybrid chitosan network structure nanocomposite scaffolds. Acta Biomater. 2011;7(9):3432-3445.

17. Suh JK, Matthew HW. Application of chitosan-based polysaccharide biomaterials in cartilage tissue engineering: a review. Biomaterials. 2000;21(24):2589-2598.

18. Ravi Kumar MNV. A review of chitin and chitosan applications. React Funct Polym. 2000;46(1):1-27.

19. Lauto A, Ohebshalom M, Esposito M, et al. Self-expandable chitosan stent: design and preparation. Biomaterials. 2001;22(13):1869-1874.

20. Tada DB, Singh S, Nagesha D, et al. Chitosan film containing poly(D,Llactic-co-glycolic acid) nanoparticles: a platform for localized dual-drug release. Pharm Res. 2010;27(8):1738-1745.

21. Khan TA, Peh KK, Ch'ng HS. Mechanical, bioadhesive strength and biological evaluations of chitosan films for wound dressing. $J$ Pharm Pharmaceut Sci. 2000;3(3):303-311.

22. Jayakumar R, Prabaharan M, Sudheesh Kumar PT, Nair SV, Tamura H. Biomaterials based on chitin and chitosan in wound dressing applications. Biotechnol Adv. 2011;29(3):322-337.

23. Mi FL, Sung HW, Shyu SS. Synthesis and characterization of a novel chitosan-based network prepared using naturally occurring crosslinker. J Polym Sci A Polym Chem. 2000;38(15):2804-2814.

24. Jin J, Song M, Hourston DJ. Novel chitosan-based films cross-linked by genipin with improved physical properties. Biomacromolecules. 2004;5(1):162-168
25. Mi FL, Tan YC, Liang HF, Sung HW. In vivo biocompatibility and degradability of a novel injectable-chitosan-based implant. Biomaterials. 2002;23(1):181-191.

26. Wang $\mathrm{G}$, Zheng $\mathrm{L}$, Zhao $\mathrm{H}$, et al. In vitro assessment of the differentiation potential of bone marrow-derived mesenchymal stem cells on genipin-chitosan conjugation scaffold with surface hydroxyapatite nanostructure for bone tissue engineering. Tissue Eng Part A. 2011;17(9-10):1341-1349.

27. Wang G, Zhao H, Miao J, et al. Construction of A fluorescent nanostructured chitosan-hydroxyapatite scaffold by nanocrystallon induced biomimetic mineralization and its cell biocompatibility. ACSAppl Mater Inter. 2011;3(5);1692-1701.

28. Pan Y, Wu T, Bao H, Li L. Green fabrication of chitosan films reinforced with parallel aligned graphene oxide. Carbohydr Polym. 2011;83(4): 1908-1915.

29. Yang X, Tu Y, Li L, Shang S, Tao XM. Well-dispersed chitosan/ graphene oxide nanocomposites. ACS Appl Mater Interfaces. 2010;2(6): 1707-1713.

30. Han D, Yan L, Chen W, Li W. Preparation of chitosan/graphene oxide composite film with enhanced mechanical strength in the wet state. Carbohydr Polym. 2011;83(2):653-658.

31. Marcano DC, Kosynkin DV, Berlin JM, et al. Improved synthesis of graphene oxide. ACS Nano. 2010;4(8):4806-4814.

32. Wang SM, Huang QZ, Wang QS. Study on the synergetic degradation of chitosan with ultraviolet light and hydrogen peroxide. Carbohydr Res. 2005;340(6):1143-1147.

33. Butler MF, Ng YF, Pudney PD. Mechanism and kinetics of the crosslinking reaction between biopolymers containing primary amine groups and genipin. J Polym Sci A Polym Chem. 2003;41(24);3941-3953.

34. Lee SW, Lim JM, Bhoo SH, Paik YS, Hahn TR. Colorimetric determination of amino acids using genipin from Gardenia jasminoides. Anal Chim Acta. 2003;480(2):267-274.

35. Yuan Y, Chesnutt BM, Utturkar G, et al. The effect of cross-linking of chitosan microspheres with genipin on protein release. Carbohydr Polym. 2007;68(3):561-567.

36. Porstmann B, Jung K, Schmechta H, et al. Measurement of lysozyme in human body fluids: comparison of various enzyme immunoassay techniques and their diagnostic application. Clin Biochem. 1989;22(5):349-355.

37. Fan H, Wang L, Zhao K, et al. Fabrication, mechanical properties, and biocompatibility of graphene-reinforced chitosan composites. Biomacromolecules. 2010;11(9):2345-2351.

38. Mi FL. Synthesis and characterization of a novel chitosan-gelatin bioconjugate with fluorescence emission. Biomacromolecules. 2005;6(2): 975-987.

39. Chen H, Ouyang W, Lawuyi B, Martoni C, Prakash S. Reaction of chitosan with genipin and its fluorogenic attributes for potential microcapsule membrane characterization. J Biomed Mater Res A. 2005;75(4):917-927.

40. Eda G, Lin YY, Mattevi C, et al. Blue photoluminescence from chemically derived graphene oxide. Adv Mater Weinheim. 2010;22(4): 505-509.

41. Loh KP, Bao Q, Eda G, Chhowalla M. Graphene oxide as a chemically tunable platform for optical applications. Nat Chem. 2010;2(12): $1015-1024$.

42. Kim J, Cote LJ, Kim F, Huang J. Visualizing graphene based sheets by fluorescence quenching microscopy. J Am Chem Soc. 2010;132(1): 260-267.

43. Wang K, Ruan J, Song H, et al. Biocompatibility of graphene oxide. Nanoscale Res Lett. 2011;6(1):8.

44. Chang Y, Yang ST, Liu JH, et al. In vitro toxicity evaluation of graphene oxide on A549 cells. Toxicol Lett. 2011;200(3):201-210. 


\section{Supplementary figures}
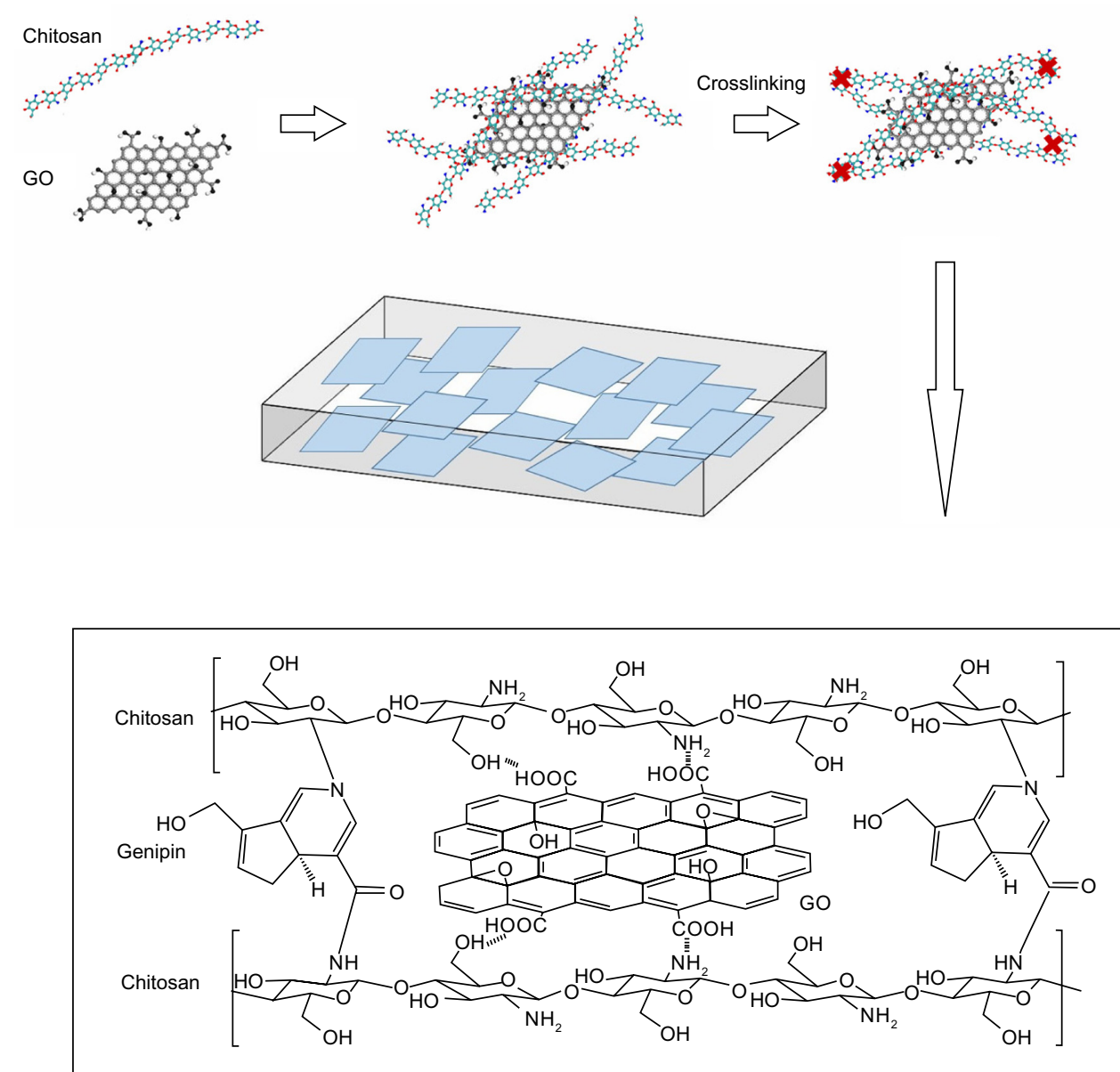

Figure SI Schematic representation of the GCS/GO composite.

Abbreviations: GCS, genipin-cross-linked chitosan; GO, graphene oxide.
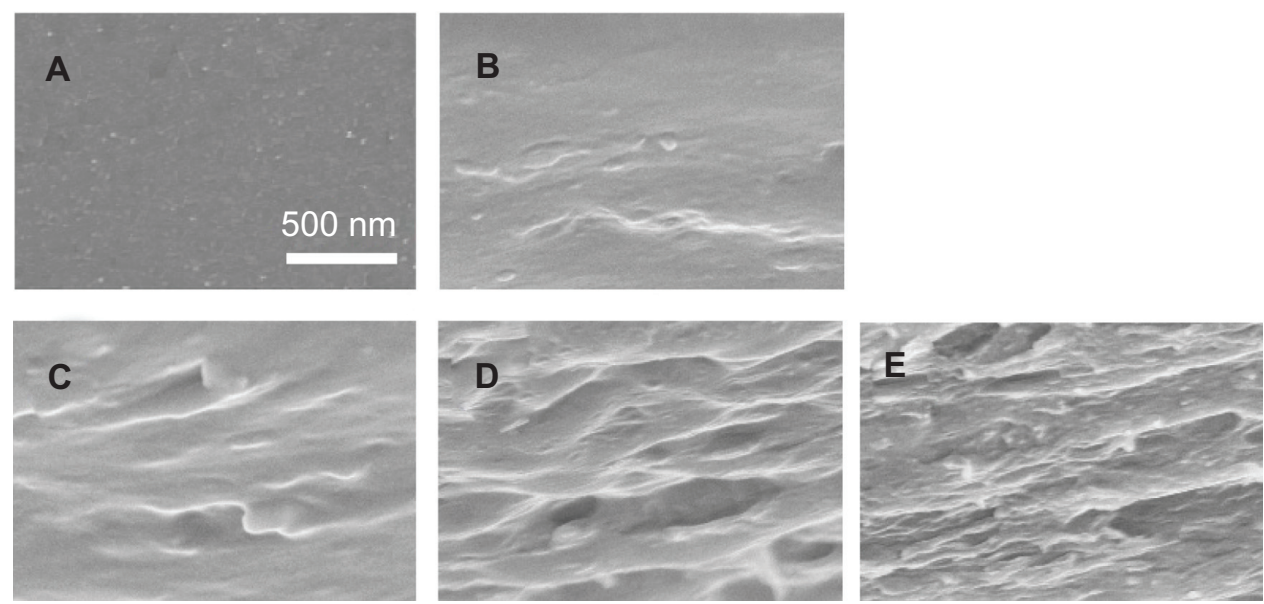

Figure S2 Cross-sectional SEM images of GCS (A), GCS/GO-0.I (B), GCS/GO-0.5 (C), GCS/GO-I (D), and GCS/GO-2 (E).

Abbreviations: GCS, genipin-cross-linked chitosan; GO, graphene oxide; SEM, scanning electron microscope. 


\section{Publish your work in this journal}

The International Journal of Nanomedicine is an international, peerreviewed journal focusing on the application of nanotechnology in diagnostics, therapeutics, and drug delivery systems throughout the biomedical field. This journal is indexed on PubMed Central, MedLine, CAS, SciSearch $\AA$, Current Contents ${ }^{\circledR} /$ Clinical Medicine,

Journal Citation Reports/Science Edition, EMBase, Scopus and the Elsevier Bibliographic databases. The manuscript management system is completely online and includes a very quick and fair peer-review system, which is all easy to use. Visit http://www.dovepress.com/ testimonials.php to read real quotes from published authors.

Submit your manuscript here: http://www.dovepress.com/international-journal-of-nanomedicine-journal 